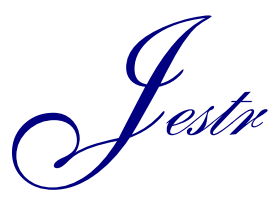

Journal of Engineering Science and Technology Review 4 (3) (2011) 227 - 232

Special Issue on Econophysics

Research Article

www.jestr.org

\title{
Nonlinear Supply And Demand Model With Two Interrelated Markets
}

\author{
L. Zachilas * and A. Gkana
}

Dep. of Economics, University of Thessaly, 43 Korai str. GR-38333, Volos, Greece.

\begin{abstract}
Our target in this paper is to observe how the presence of nonlinear terms in the supply and demand model changes the price behavior of the system. The dynamical analysis refers, mainly, to discrete dynamical systems. We start with a simple linear supply and demand model with two markets interacting with each other. The stability conditions for the linear model are presented. Afterwards we add nonlinear terms and try to observe, if the presence of nonlinearity in the supply and demand model with two interrelated markets exhibits chaotic price behavior. Chaos bursts in the system via period-doubling bifurcations, and all orbits converge to a strange attractor.
\end{abstract}

Keywords: Chaos, Strange attractor, Non-linear supply and demand model, Bifurcations.

\section{Introduction}

The simplest supply and demand (S-D) modelin discrete time is the linear cobweb model [1], which explains the cyclical tendencies that we observe in a lot of commodity markets. Following the static expectations scheme, the expected price in period $t$ is equal to (and determined by) the price of the previous period (suppliers have naïve expectations for prices). In this case we have a linear first order difference equation. There are many extensions of this model such as the cobweb model with alternative assumption in accordance with the expected price in period $t$. The period $t$ is an adjustment of the forecast error in the previous guess [2]. Another alternative assumption is when the expected price is determined by the price of the two previous periods [3], where we have a second order difference equation (a two-period lag in supply). As for the case of discrete dynamical systems a simple linear model is the corn - hog cycle mentioned by Ezekiel [1] and Waugh [4], which refers to two markets interacting each other. This model can be applied in all cases of an animal - feed interaction. All these linear supply and demand models are good, but they can only give stable or unstable fixed points or even periodic cycles, while the price behavior in realworld systems is usually much more complicated. On the other hand, nonlinearities in supply and demand [5-7], may give chaotic price dynamics for different producers' adaptive expectations schemes. For example, the nonlinear version of the cobweb model with adaptive expectations [8] shows that under simple and reasonable economic assumptions chaos may occur in the system. Another case is the nonlinear model mentioned by Dieci and Westerhoff $[9,10]$. According to this model, market interactions may change the cyclical

\footnotetext{
*E-mail address: zachilas@uth.gr
}

ISSN: 1791-2377 @ 2011 Kavala Institute of Technology. All rights reserved. behavior of prices captured by the classical cobweb model and drive to instability and complexity. Also Currie and Kubin [11], Hommes \& van Eekelen [12], and Yousefi et al. [13] have shown how small interdependencies, "linked" from the demand side, can dramatically change the dynamics for interdependent cobweb economies. Finally another example is the nonlinear cobweb model mentioned by Onozaki, Sieg \& Yokoo [14], in which we have two types of producers (cautious adapters and naïve optimizers). In such a market with cautious adapters and a single naïve optimizer may appear many coexisting periodic attractors.

The main target in this paper is to observe how the presence of nonlinearity changes the behavior of the solutions. For our analysis we will use the animal-feed interaction model, because the assumption of two markets is much more realistic from the case of only one market, while at the same time is simple as much as possible. So we briefly begin with the linear animal-feed interaction model and then we introduce nonlinearity in the system in order to observe the way the price behavior of the system changes.

\section{The Linear S-D Model}

According to the corn-hog cycle mentioned by Ezekiel [1] and Waugh [4], the animal - feed interaction model consists of two markets:

$$
\begin{aligned}
& \text { Corn Market: } \begin{cases}d_{t}^{c}=a_{1}-b_{1} p_{t}^{c} & , b_{1}>0 \\
s_{t}^{c}=c_{1}+d_{1} p_{t-1}^{c} & , d_{1}>0\end{cases} \\
& \text { Hog Market: } \begin{cases}d_{t}^{h}=a_{2}-b_{2} p_{t}^{h} & , b_{2}>0 \\
s_{t}^{h}=c_{2}+d_{2} p_{t-1}^{h}+e p_{t-1}^{c}, d_{2}>0, e<0\end{cases}
\end{aligned}
$$


where, $d_{t}^{c}, s_{t}^{c}$ : are the demand and supply functions for corn, and $d_{t}^{h}, s_{t}^{h}$ :are the demand and supply functions of hogs. The model used in both markets is the simple linear cobweb model $^{1}$ [1]. Following the static expectations scheme, the expected price (of corn, $p_{t}^{c^{e}}$, and hogs, $p_{t}^{h^{e}}$ ) in period $t$ is equal to (and determined by) the price of the previous period, $p_{t}^{c^{e}}=p_{t-1}^{c}$ and $p_{t}^{h^{e}}=p_{t-1}^{h}$. However, while the supply of corn $s_{t}^{c}$ is determined only by $p_{t-1}^{c}$, the supply of hogs $s_{t}^{h}$ depends on both $p_{t-1}^{h}$ and $p_{t-1}^{c}$, since corn is the main food of hogs. For this reason, the parameter takes only negative values, since an increase in the corn price makes the food of hogs more expensive, so that the supply of the hogs decreases.

Substituting the demand and supply equations into the equilibrium condition, $d_{t}^{c}=s_{t}^{c}$ and $d_{t}^{h}=s_{t}^{h}$, we obtain the system of two linear non-homogeneous first order difference equations:

$$
\left\{\begin{array}{l}
p_{t}^{c}=\frac{a_{1}-c_{1}}{b_{1}}-\frac{d_{1}}{b_{1}} p_{t-1}^{c} \\
p_{t}^{h}=\frac{a_{2}-c_{2}}{b_{2}}-\frac{d_{2}}{b_{2}} p_{t-1}^{h}-\frac{e}{b_{2}} p_{t-1}^{c}
\end{array}\right.
$$

The fixed point in the corn and hog market is:

$$
\left(p_{c}^{*}, p_{h}^{*}\right)=\left(\frac{a_{1}-c_{1}}{b_{1}+d_{1}}, \frac{a_{2}-c_{2}}{b_{2}+d_{2}}-\frac{e \cdot\left(a_{1}-c_{1}\right)}{\left(b_{2}+d_{2}\right) \cdot\left(b_{1}+d_{1}\right)}\right)
$$

It is known that, in systems with real nonequal eigenvalues, the equilibrium is stable, if the roots of the characteristic equation are within the unit circle [15]. Thus the stability condition for the corn market is: $\left|-d_{1} / b_{1}\right|<1$. The stability condition in the hog market, assuming that the corn price is constant, is: $\left|-d_{2} / b_{2}\right|<1$. Consequently, if $\left|b_{1}\right|>d_{1}$ and $\left|b_{2}\right|>d_{2}$, the solutions for both markets $p_{t}^{c}, p_{t}^{h}$, for any initial values, $p_{0}^{c}, p_{0}^{h}$, will tend toward the fixed points $p_{c}^{*}, p_{h}^{*}$ respectively.

Using the following values for the parameters [15]:

$a_{1}=24, b_{1}=5, c_{1}=-4, d_{1}=2, a_{2}=20, b_{2}=5$, $c_{2}=2.5, d_{2}=2.5$ and $e=-2$

the system of the two difference equations becomes:

$\left\{\begin{array}{l}p_{t}^{c}=5.6-0.4 p_{t-1}^{c} \\ p_{t}^{h}=3.5-0.5 p_{t-1}^{h}+0.4 p_{t-1}^{c}\end{array}\right.$

The unique fixed point of the system is: $\left(p_{c}^{*}, p_{h}^{*}\right)=(4,3.4)$.

The roots of the characteristic equation are $\lambda_{1}=-0.4$ and $\lambda_{2}=-0.5$, which means that the eigenvalues of the system are within the unit circle, $\left(\left|\lambda_{1}\right|=0.4<1\right.$ and $\left.\left|\lambda_{2}\right|=0.5<1\right)$ and the fixed points for

\footnotetext{
${ }^{1}$ According to the cobweb model [1], the supply function is given by: $q_{t}^{s}=c+d p_{t}^{e}$, with $p_{t}^{e}=p_{t-1}$
}

both markets are stable.

In Fig. 1(a), we depict the plot $\left(p_{t}^{h}, p_{t}^{c}\right)$, while in Fig. 1(b), we can see the behavior of the solutions $\left(t, p_{t}^{h}\right)$ and $\left(t, p_{t}^{c}\right)$, as $t$ tends to infinity. In both figures we give the first 30 consecutive terms of the sequence, while the initial conditions are $p_{0}^{h}=p_{0}^{c}=0.5$. In Fig. 1(b), we can easily observe that $p_{t}^{h} \rightarrow p_{h}^{*}=3.4$ and $p_{t}^{c} \rightarrow p_{c}^{*}=4$. So, as it was expected ${ }^{2}$, we observe that, when the slope of the supply curves for both markets is less than the slope of the demand curve, (i.e. $\left|b_{1}\right|>d_{1}$ and $\left|b_{2}\right|>d_{2}$ ), then, for any initial value, all solutions tend toward the fixed point, which is an attractor.

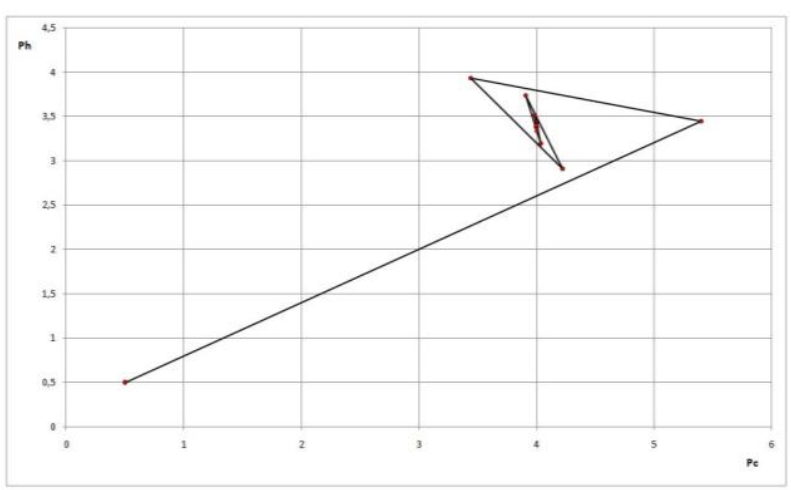

Fig. 1(a). The first 30 consequents of the graph $\left(p_{t}^{c}, p_{t}^{h}\right)$.

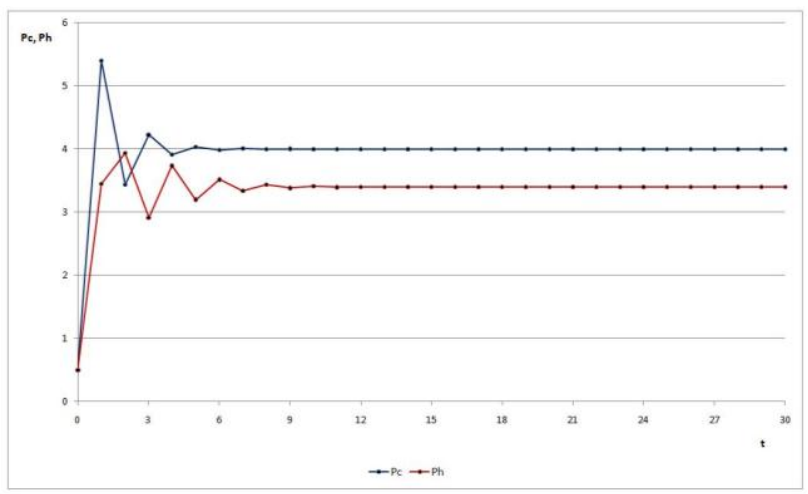

Fig. 1(b). Time series $\left(t, p_{t}^{h}\right)$ and $\left(t, p_{t}^{c}\right)$, initial conditions $p_{0}^{h}=p_{0}^{c}=0.5$, with $\left|b_{1}\right|>d_{1}$ and $\left|b_{2}\right|>d_{2}$.

However, the real-world systems are more complicated and, mainly, are not linear. Thus, it would be really interesting to study the qualitative and quantitative change in case that the supply and demand model with two interrelated markets becomes nonlinear. In order to make our analysis as simple as possible, we use the nonlinear form of the cobweb model $^{3}$ [15], bin which the supply function is nonlinear at price.

\section{The Nonlinear S-D Model}

${ }^{2}$ The stability of the equilibrium price in the cobweb model [1] also depends on the ratio of the slopes of supply and demand curves.

${ }^{3}$ According to the nonlinear cobweb model [14] the supply function is given by $q_{t}^{s}=c+d p_{t-1}^{2}$. 
In order to detect, if the nonlinearity in the corn market changes the price behavior in both markets, let us assume that the nonlinear supply function is: $s_{t}^{c}=c_{1}+d_{1} \cdot\left(p_{t-1}^{c}\right)^{2}$.

In this case the nonlinear system of the two nonhomogenous first-order difference equations is given by:

$$
\left\{\begin{array}{l}
p_{t}^{c}=\frac{a_{1}-c_{1}}{b_{1}}-\frac{d_{1}}{b_{1}} \cdot\left(p_{t-1}^{c}\right)^{2} \\
p_{t}^{h}=\frac{a_{2}-c_{2}}{b_{2}}-\frac{d_{2}}{b_{2}} \cdot p_{t-1}^{h}-\frac{e}{b_{2}} p_{t-1}^{c}
\end{array}\right.
$$

Thus, we will try to see if shifts in the demand curve of the corn market, represented by a variation in the parameter $a_{1}$, change the characteristics of the fixed point of the system in both markets. In order to study the new model, we will vary parameter $\mathrm{a}_{1}$, while the values of all the rest parameters will be "frozen".

For simplicity and comparability reasons, we keep the same values for the parameters that we used in Section 1 for the linear model. The nonlinear discrete system becomes:

$$
\left\{\begin{array}{l}
p_{t}^{c}=\frac{a_{1}+4}{5}-0.4 \cdot\left(p_{t-1}^{c}\right)^{2} \\
p_{t}^{h}=3.5-0.5 p_{t-1}^{h}+0.4 p_{t-1}^{c}
\end{array}\right.
$$

By giving different negative and positive values in the parameter $a_{1}$, we can distinguish the successive changes in the behavior of solutions. A good way to summarize these changes is to plot the bifurcation diagram $\left(a_{1}, p_{c}^{*}\right)$, as the parameter varies in the interval $-7.125 \leq a_{1} \leq 21$ (Fig. 2). The initial conditions of the orbits are $p_{0}^{h}=p_{0}^{c}=0.5$. A bifurcation diagram shows the nature of the fixed point changes as the value of the parameter change [16].

Specifically:

$>$ For $a_{1}<-7.125$, the system has no real fixed points and all orbits, for every initial condition, tend to infinity.

$>$ At $a_{1} \approx 7.125$, a single neutral fixed point appears in the system at $p_{c}^{*}=-1.25$. The derivative at the fixed point is equal to 1 . Thus, when $p_{0}^{c}>-1.25$, the solutions converge to the fixed point, while when $p_{0}^{c}<-1.25$, the solutions diverge from the fixed point, as $t$ increases.

$>$ For $a_{1}>-7.125$, a pair of fixed points $p_{c_{1}}^{*}<0, p_{c_{2}}^{*}<0$ appears in the system. The one is attracting and the second is repelling. As the value of the parameter increases, the two fixed points move away from each other and the attracting fixed point becomes positive. The phenomenon is called "saddle node bifurcation" [17].

$>$ For $a_{1}>5.35$, the positive fixed point changes from attracting to repelling and all orbits lie on an attracting 2 - period cycle. This is the case of "period doubling bifurcation" [17].

$>$ For $a_{1}>11.65$, the attracting 2 - period cycle becomes repelling and a new double periodic attracting cycle is born. So all orbits lie on a 4 - period cycle around the positive fixed point.
$>$ For $a_{1}>13.15$ : The attracting 4 - period cycle becomes repelling and a new (double period) attracting 8 period cycle is born.

$>$ For $a_{1}>13.45$, the process of period doubling bifurcations continues with the births of new attracting period cycles (16-period cycle, 32-period cycle, 64period cycle, and so on).

$>$ For $a_{1}>13.55$, the successive period doubling bifurcations lead eventually to a situation where the behavior of solutions is very complex. Thus, although the system is deterministic, the behavior of solutions appears as random. This is what Devaney [18] called "deterministic complexity on the verge of randomness". So in this case, we encounter a new type of behavior, which is called "chaos". At this point we observe the phenomenon "period - doubling route to chaos" [17].

$>$ For $a_{1}>17.95$, and for a small interval of the varying parameter, the behavior of solutions changes back to non-chaotic and all orbits converge to an attracting 3 period cycle.

$>$ For $a_{1}>18.15$, the attracting 3 - period cycle becomes repelling and a new attracting 6 - period cycle is born. This scenario is repeated $(6$ - period cycle, 12 - period cycle...) and once more the period - doubling route to chaos takes place.

$>$ For $a_{1}>21$, all solutions for every initial condition tend to infinity.

So, the qualitative results that come from the bifurcation diagram are: (a) all orbits initially tend toward an attracting positive fixed point, (b) for $a_{1}>5.35$, and at specific values of the varying parameter, all orbits lie on attracting 2 period cycle, 4 - period cycle, 8 - cycle, and so on, and (c) chaos is reached through period doubling bifurcations.

Moreover, if we look carefully at the bifurcation diagram, we observe that among the chaotic regions, for some specific values of $a_{1}$, the orbits lie on integer-period cycles $(6$ - period, 5 - period, 3 - period). In explicitly, there are windows with periodic orbits that are surrounded by chaotic behavior.

The above analysis was done for the case that the slope of the supply curve is less than the slope of the demand curve, $\left|b_{1}\right|=5>d_{1}=2$. However, we have encountered the same behavior of solutions for each slope of supply curve $d_{1}$ (i.e. for the other two cases, $\left|b_{1}\right|=d_{1}$ and $\left|b_{1}\right|<d_{1}$ ). It is worthy of note that the same qualitative behavior was encountered in the study of the hog market. This is true, since the price of the hog market depends on the price of the corn market.

Thereby we observe that in contradiction to the linear model, even in this simple nonlinear model, shifts in the demand curve of the corn market may not only change the behavior of solutions, but they can also exhibit a chaotic price behavior. This is really important because if such a simple nonlinear form exhibits a chaotic price behavior, more complex nonlinear forms (that represent the real market) will exhibit even more chaotic results.

The fact that shifts in the demand curve lead to the appearance of chaos implies that our system is sensitive to initial conditions. Sensitive dependence on initial conditions means that small deviations in the initial values can lead to radical changes to the orbit of the system [15]. 


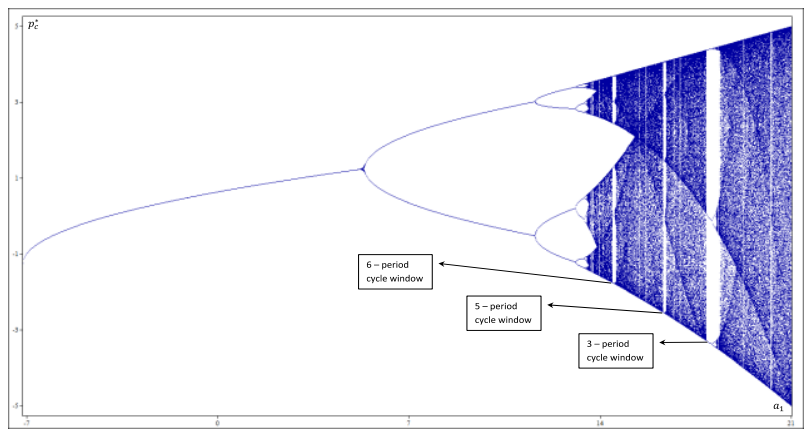

Fig. 2. The bifurcation diagram $\left(a_{1}, p_{c}^{*}\right)$ in the interval $-7.125 \leq a_{1} \leq 21$. The initial conditions were the same for all orbits $\left(p_{0}^{h}=p_{0}^{c}=0.5\right)$.

A good proof is the draw of the solution graphs for two neighboring initial conditions, $p_{0}^{c}=0.5$ and $p_{0}^{c}=0.499$, for $T=70$ periods. We have chosen a "chaotic" value of the parameter $\left(\mathrm{a}_{1}=15.5\right)$ (Fig.3). The two orbits are identical in the first 20 periods (approximately). Afterwards, they start to deviate considerably. The sensitivity of initial conditions is really important. The inability to determine the initial conditions with infinite precision makes the system quite unpredictable, at least for long periods [17].

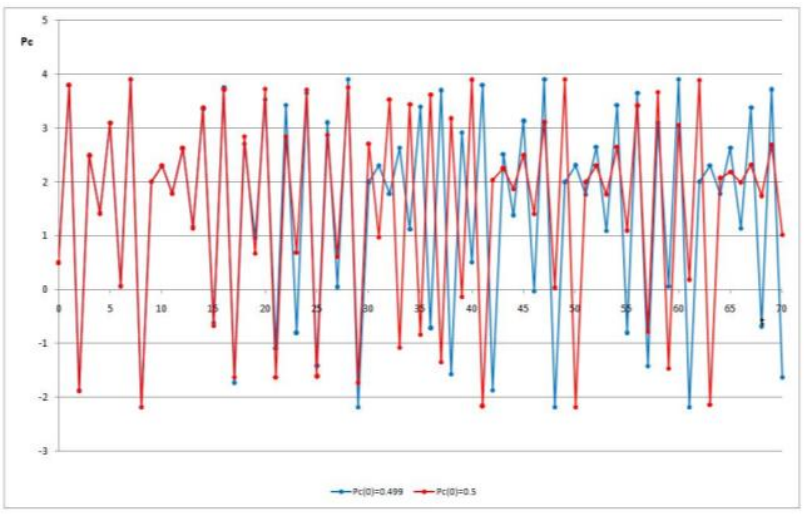

Fig. 3. Time series $\left(t, p_{t}^{c}\right)$ for two orbits with initial conditions $p_{0}^{c}=0.5$ (in red) and $p_{0}^{c}=0.499$ (in blue). Parameter $a_{1}$ was chosen in the chaotic region and has the value of 15.5

Up to now, we found a rather expected ${ }^{4}$ phenomenon. The presence of nonlinearity in the model of supply and demand with two interrelated markets can exhibit a chaotic price behavior. However, $b_{y}$ plotting the phase diagram $\left(p_{t}^{c}, p_{t}^{h}\right)$ and choosing a "chaotic" value of the parameter (e.g. $a_{1}=15.5$ ), we see something peculiar (Fig.4). Normally in chaotic regions, the successive iterations in the graph $\left(p_{t}^{c}, p_{t}^{h}\right)$ would have filled the whole map. However, what we actually observe is that for every initial value $p_{0}^{c}, p_{0}^{h}$, all solutions tend to this strange orbit. Since (i) all solutions tend to it and (ii) this orbit is very different from the simpler attractors, (i.e. it is neither an attracting fixed point nor a periodic orbit), it is called "strange attractor". For all the initial conditions, all solutions converge to this

${ }^{4}$ The supply function of this model has the same nonlinear form as the supply function of the nonlinear cobweb model [15] in which shifts in the demand curve (variations in the parameter $a$ ) exhibit also chaotic price behavior. strange attractor, each one in a different way. Thus, this strange attractor is also a chaotic one [15].

In Fig. 5, we highlight the deeper and deeper magnifications; the initial geometric shape repeats itself inside the next zoom level. This feature of this strange orbit is called "self - similarity" and all geometric shapes with this feature are called fractals [16]. The fractal structure is the main feature of strange attractors. The existence of a strange attractor is really important, because it indicates the existence of a hidden fine structure into chaos. So, although the price behavior is chaotic, all solutions end up converging to this strange attractor.

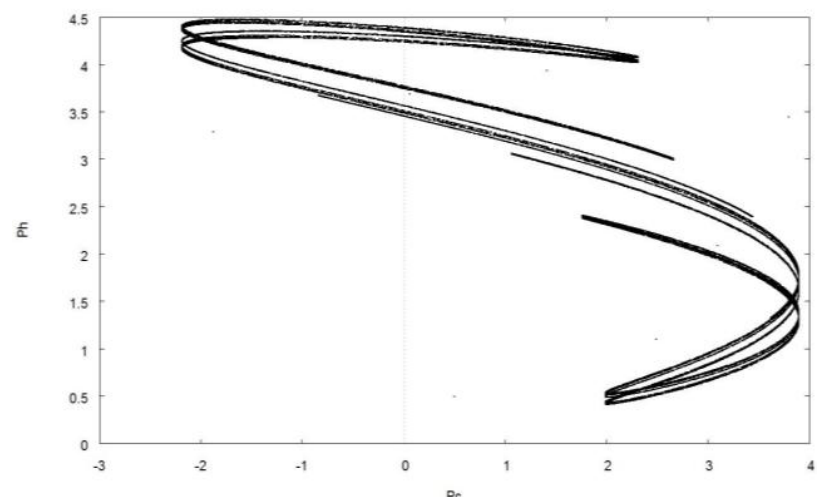

Fig. 4. The phase diagram $\left(p_{t}^{c}, p_{t}^{h}\right)$ with initial conditions $p_{0}^{h}=p_{0}^{c}=0.5$. Parameter $a_{1}=15.5$ (in the chaotic region) leads to a strange attractor. All solutions, and for every initial conditions, tend to this strange orbit.

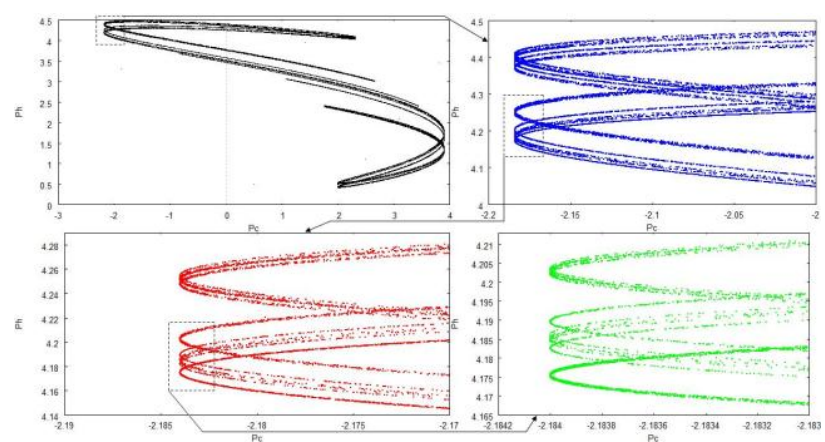

Fig. 5. The phase diagram $\left(p_{t}^{c}, p_{t}^{h}\right)$ with initial conditions $p_{0}^{h}=p_{0}^{c}=0.5$ $\left(a_{1}=15.5\right)$ in different zoom levels. In particular, we point out the selfsimilarity of the marked orthogonal region. The strange attractor repeats itself in the first zoom (in blue), in the second zoom (in red) and in the third zoom (in green). These geometric shapes are called fractals.

We saw that the orbits (illustrated in Fig. 3) remain close to each other only for the first periods. After a short period they diverge from each other drastically. One way of measuring the divergence between these two orbits is the Lyapunov exponents [19]. Positive Lyapunov exponents indicate that the two orbits diverge from one another exponentially. While negative Lyapunov exponents indicate that the orbits converge to each other [15]. The existence of a single positive Lyapunov exponent implies the chaotic behavior of the system and consequently the sensitive dependence on initial conditions. Finally, the higher the value of a Lyapunov exponent, the lower the possibility of 
predictability. We plot the maximum Lyapunov ${ }^{5}$ exponent diagram, $\left(a_{1}, L\right)$, for different values of the parameter in the interval $0 \leq a_{1} \leq 21$, along with the bifurcation diagram (Fig.6).

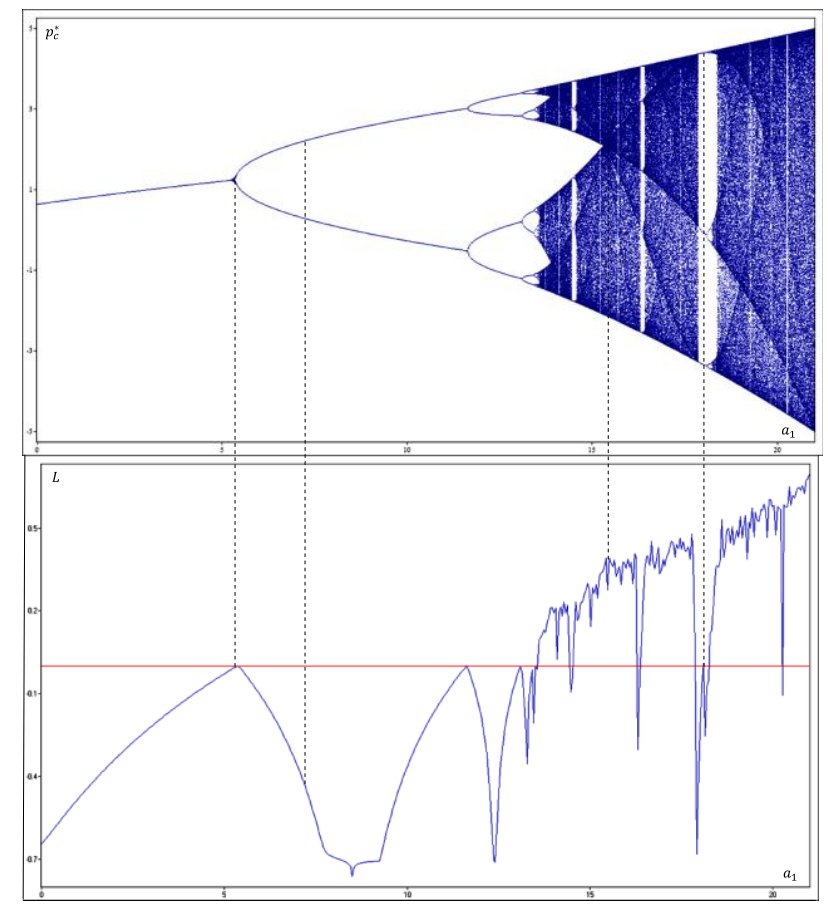

Fig. 5. The maximum Lyapunov exponent along with the bifurcation diagram. The initial conditions were the same for all orbits $\left(p_{0}^{h}=p_{0}^{c}=0.5\right)$. The parameter was varied in the interval $0 \leq a_{1} \leq 21$.

In Fig.6, we observe that:

$>$ For those values of the parameter that we have non-chaotic behavior, the Lyapunov exponent is negative, $L\left(a_{1}\right)<0$, and the orbits always converge to each other. The predictability of the behavior of the system is attainable for an infinite period of time.

$>$ On the other hand, for those parameter values for which the behavior of solutions is chaotic, the Lyapunov exponent is positive, $L\left(a_{1}\right)>0$, and the two orbits diverge from each other exponentially. In this case the prediction of future states of the system, after a short span of time, becomes impossible.

$>$ While, those values of the parameter, on which bifurcations occur in the system, correspond to a zero Lyapunov exponent, $L\left(a_{1}\right)=0$.

Finally, from the Lyapunov exponent diagram we see that the maximum value of the Lyapunov exponent is $L \approx 0.72$ for $a_{1} \approx 21$. This implies that, for this value of the parameter, we have less possibility of predictability for the system.

\section{Conclusions}

We have seen that the presence of nonlinearity in the model of supply and demand with two interrelated markets can exhibit a chaotic price behavior. The system we examined is deterministic - described by specific difference equations and the predictions are possible for an infinite period of time, only when the parameter varies in a specific interval. However, when the varying parameter takes higher values, chaos appears. Chaos implies the sensitive dependence on initial conditions . The inability to determine the initial conditions with infinite precision has as a result the predictability for long periods to be impossible

${ }^{5} \mathrm{We}$ have used the software package E\&F Chaos that uses the Wolf method [19] in order to calculate the largest Lyapunov exponent [20]. 


\section{References}

1. M. Ezekiel, The Cobweb Theorem, The Quarterly Journal of Economics, 52255 (1938)

2. M. Nerlove, Adaptive Expectations and Cobweb Phenomena, The Quarterly Journal of Economics, 72227 (1958).

3. M. R. Goodwin, Dynamical Coupling with Especial Reference to Markets Having Production Lags, Econometrica, 15181 (1947).

4. F. V. Waugh, Cobweb models, Journal of Farm Economics, 46 (4) $732(1964)$

5. C. Chiarella, The cobweb model, its instability and the onset of chaos, Economic Modelling, 5377 (1988).

6. R. H. Day, Complex economic dynamics: an introduction to dynamical systems and market mechanisms, MIT Press, Cambridge, MA, (1994).

7. C. H. Hommes, "On the consistency of backward-looking expectations: the case of the cobweb", Journal of Economic Behavior and Organization, 33, 333 (1998).

8. C. H. Hommes, Dynamics of the cobweb model with adaptive expectations and non-linear supply and demand, Journal of Economic Behavior and Organization, 24315 (1994).

9. R. Dieci, F. Westerhoff, Stability analysis of a cobweb model with market interactions, Applied Mathematics and Computation, 215 2011 (2009).

10. R. Dieci, F.Westerhoff, Interacting Cobweb Markets, Journal of Economic Behavior \& Organization, 75461 (2010).
11. M.Currie, I. Kubin, Non-linearities and partial analysis, Economic Letters, 4927 (1995).

12. C. H. Hommes, A. van Eekelen, Partial equilibrium analysis in a noisy chaotic market, Economics Letters, 53275 (1996).

13. S. Yousefi, Y. Maistrenko, S.Popovych, Complex dynamics in a simple model of interdependent open economies, Discrete Dynamics in Nature and Society, 5161 (2000).

14. T. Ozonaki, G. Sieg, M. Yokoo, Stability, chaos and multiple attractors: a single agent makes a difference, Journal of Economic Dynamics \& Control, 271917 (2003).

15. R. Shone, Economic Dynamics: Phase Diagrams and their Economic Application, Cambridge University Press (2002).

16. L.R. Devaney, W.M. Hirsch, S. Smale, Differential Equations, Dynamical Systems \& An Introduction to Chaos, Elsevier Academic Press (2004).

17. P. Blanchard, L.R. Devaney, R.G. Hall, Differential Equations, Thomson Brooks/Cole (2006)

18. L. R. Devaney, An Introduction to Chaotic Dynamical Systems, 2nd edition, New York, Addison-Wesley (1989).

19. P. J. Van der Weele, Chaos and Fractals. University of Patras. Department Mathematics.(http://www.math.upatras.gr/ weele/files/ChaosAnd FractalsFyllo\%20\%282007-08\%29.pdf) (2007)

20. A. Wolf, J. B. Swift, L. Swinney, J. A. Vastano, Determining Lyapunov exponents from a timeseries Physica D, 16285 (1985). 